\title{
Rapid progression and recovery of massive gastric mucosal damage complicated by Henoch-Schönlein purpura in a patient with liver cirrhosis: A case report
}

\author{
TAE-GEUN GWEON and JUNG HYUN KWON
}

\begin{abstract}
Division of Gastroenterology and Hepatology, Department of Internal Medicine, Incheon St. Mary's Hospital, College of Medicine, The Catholic University of Korea, Incheon 21431, Republic of Korea
\end{abstract}

Received December 25, 2018; Accepted July 5, 2019

DOI: $10.3892 /$ etm.2019.7815

\begin{abstract}
Endoscopic findings associated with gastric ischemia include friable gastric mucosa, irregular ulceration, petechiae and purpura. The etiology of gastric ischemia includes shock, sepsis, thrombosis, embolism and vasculitis. Henoch-Schönlein purpura (HSP) is a leukocytoclastic vasculitis that affects small vessels in the skin, kidney and gastrointestinal (GI) tract. Rapid progression of HSP manifested with acute gastric mucosal bleeding has been rarely reported. The present case study reports on a patient who had undergone endoscopic variceal ligation for esophageal varix bleeding and the gastric mucosa exhibited no evidence of HSP. After 9 days, reexamination using endoscopy to check for new-onset hematemesis revealed massive necrosis in the gastric mucosa, friable mucosal tissue and acute ischemic GI bleeding. The patient was treated with corticosteroid injections for 7 days, which caused a marked regression of the hemorrhagic gastric mucosa. The present case study reported on this patient with acute GI bleeding caused by HSP who was successfully treated with corticosteroids.
\end{abstract}

\section{Introduction}

Henoch-Schönlein purpura (HSP) is a type of leukocytoclastic vasculitis that affects small vessels of the skin, kidney and gastrointestinal (GI) tract. The classic triad of HSP symptoms includes purpura, arthritis and abdominal pain (1). Deposition of immune cells causes mucosal ischemia in the affected organs (2). The GI tract is involved in 50-60\% of patients (3). Colicky abdominal pain, vomiting and GI bleeding are

Correspondence to: $\mathrm{Dr}$ Jung Hyun Kwon, Division of Gastroenterology and Hepatology, Department of Internal Medicine, Incheon St. Mary's Hospital, College of Medicine, The Catholic University of Korea, 56 Dongsu-ro, Bupyeong-gu, Incheon 21431, Republic of Korea

E-mail: doctorkwon@catholic.ac.kr

Key words: hemorrhage, gastrointestinal, Henoch-Schönlein purpura, gastric ischemia, corticoisteroid the predominant features of GI involvement. Although GI bleeding is usually occult, $30 \%$ of patients have melena or hematochezia (1). However, the rapid progression of GI bleeding within several days associated with HSP has rarely been reported (4). Although the endoscopic diagnosis of HSP is challenging, the diagnosis should be made early to prevent a poor or fatal outcome. Corticosteroids are the first-line treatment for GI bleeding complicated by HSP and have a favorable treatment efficacy $(5,6)$.

The present study reports on a rare case of acute exacerbation of HSP manifested as massive gastric mucosal damage. The patient returned for examination for ischemic GI bleeding 9 days after endoscopic variceal ligation (EVL) for esophageal varix bleeding and exhibited no evidence of HSP in the gastric mucosa. Reexamination using endoscopy to check for new-onset hematemesis, performed on the day of readmission, revealed massive submucosal hemorrhage and purpura in the gastric mucosa and multiple ulcers in the duodenum that caused acute ischemic GI bleeding. After through consideration corticosteroid was used with close monitoring, as in cases of misdiagnosis, its use may aggravate GI diseaes and thereby lead to a fatal outcome. However, this patient exhibited a favorable response to corticosteroid.

\section{Case report}

A 42-year-old male with diabetes and alcoholic liver cirrhosis visited Incheon St. Mary's Hospital (Incheon, Korea) with complaints of nausea, abdominal pain and hematemesis. The patient's initial vital signs were as follows: Blood pressure, 90/60 $\mathrm{mmHg}$; pulse rate, $115 / \mathrm{min}$; and body temperature, $37.2^{\circ} \mathrm{C}$. The patient's abdomen was distended and veins were visible. Multiple petechiae were present on the hands and lower legs, which had developed 8 months previously (Fig. 1). The laboratory results were as follows: Hemoglobin, $11.1 \mathrm{~g} / \mathrm{dl}$ (normal range, 13-18 g/dl); platelet count, 137x10 $/ 1$ (normal range, [150-450] $\mathrm{x} 10^{9} / 1$ ); white blood cell count, $22,980 / \mathrm{mm}^{3}$ (normal range,

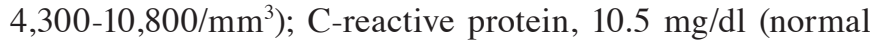
range, $<5 \mathrm{mg} / \mathrm{dl}$ ); albumin, $2.2 \mathrm{~g} / \mathrm{dl}$ (normal range, 3.4-5.4 g/dl); and creatinine, $1.9 \mathrm{mg} / \mathrm{dl}$ (normal range, $0.6-1.2 \mathrm{mg} / \mathrm{dl}$ ). Urine analysis revealed a large amount of red blood cells and proteinuria $(+++)$. At nine days prior to presentation, the patient had 
received EVL for the treatment of esophageal varix bleeding and was discharged 4 days after the EVL. During the previous admission, skin biopsies of the patient's lower leg and renal biopsy were performed for the diagnosis of vasculitis. At the time of EVL, mucosal edema and hyperemia were noted in the stomach corpus. However, there was no definite evidence of bleeding in the gastric mucosa. At this admission (9 days after EVL), a reexamination of the patient using esophagogastroduodenoscopy (EGD) revealed a post-EVL ulcer in the lower esophagus without evidence of acute variceal bleeding. However, the gastric mucosa was friable and covered with severe petechiae and purpura (Fig. 2). Multiple ulcers with diffuse, multifocal blood oozing were also observed in the stomach and duodenal bulb. Forceps biopsies were obtained from the duodenal ulcer and the purpura in the stomach corpus. The endoscopic findings were compatible with severe gastric ischemia.

Pharmacological treatment with a broad-spectrum antibiotic and proton pump inhibitor with a coating agent were initiated. The serum creatinine concentration decreased to $1.6 \mathrm{mg} / \mathrm{dl}$ at 1 day after admission. Abdominal computed tomography (CT) revealed a large volume of ascites and venous engorgement near the esophagus. The CT scan revealed no occlusion of major vessels or bowel target signs (Fig. 3). Even after 4 days of general treatment for the gastric ulcer, melena and abdominal pain remained. The second EGD revealed broad mucosal friability, purpura and blood oozing from the proximal antrum to the cardium. The serum IgA concentration was $828.0 \mathrm{mg} / \mathrm{dl}$ (normal range, $70-400 \mathrm{mg} / \mathrm{dl}$ ). Testing for antinuclear antibody, antimitochondrial antibody, antiglomerular basement membrane antibody and antineutrophil cytoplasmic antibodies were negative.

Regarding the diagnosis, it was indicated that the GI bleeding was associated with HSP after exclusion of vessel occlusion and infectious causes. Intravenous methylprednisolone was administered at a dose of $1 \mathrm{mg} / \mathrm{kg}$ once daily. At one day after the start of the corticosteroid therapy, the patient's abdominal pain was slightly improved. A follow-up EGD at 1 week after the initiation of corticosteroid treatment revealed marked regression of the petechiae and purpura. The duodenal ulcer was completely healed (Fig. 4). The laboratory results on the day of the follow-up EGD were as follows: Hemoglobin, $12.4 \mathrm{~g} / \mathrm{dl}$; platelet count, 196×10\%/1; white blood cell count, $16,210 / \mathrm{mm}^{3}$; C-reactive protein, $2.2 \mathrm{mg} / \mathrm{dl}$; albumin, $3.6 \mathrm{~g} / \mathrm{dl}$; and creatinine, $1.3 \mathrm{mg} / \mathrm{dl}$. The antibiotic treatment was stopped. The patient was discharged from hospital on day 9 and given oral prednisone and a proton pump inhibitor. Analysis of a biopsy specimen of the skin lesion indicated leukoclastic vasculitis; however, analysis of an endoscopic biopsy specimen did not provide any evidence of leukoclastic vasculitis. Helicobacter pylori was not detected on Giemsa staining. The results of the renal biopsy were compatible with diabetic nephropathy and leukoclastic vasculitis was not diagnosed.

\section{Discussion}

The present study reported on a case with rapid progression of HSP, which presented as acute ischemic GI bleeding. To the best of our knowledge, this is the first case report of acute exacerbation of HSP manifested as massive gastric mucosal damage. The patient had received EVL and the gastric mucosa
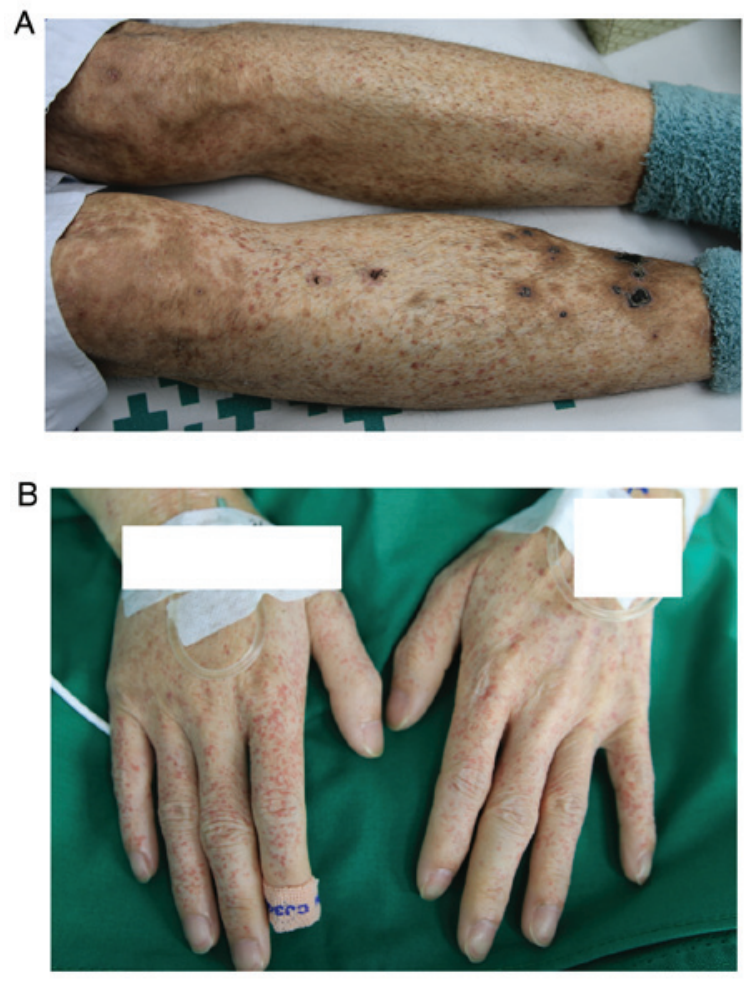

Figure 1. Multiple petechiae on (A) lower legs and (B) hands.

exhibited no evidence of bleeding at that time. At 9 days after EVL, the newly developed gastric mucosal damage and skin purpura were compatible with the diagnosis of HSP. The decision to administer corticosteroid was made after thorough consideration, because an analysis of endoscopic biopsy specimen did not provide any evidence of leukoclastic vasculitis. The initial EGD exhibited friable gastric mucosa, irregular ulceration, petechiae and purpura in the entire stomach. These endoscopic findings are common in patients with gastric ischemia (7). The etiology of gastric ischemia includes shock, sepsis, thrombosis, embolism and vasculitis (7). The gastric mucosa did not exhibit any evidence of bleeding at the time of the previous EGD 9 days previously, so the gastric ischemic must have developed rapidly. The entire gastric mucosa was inflamed, and major vessel occlusion and infectious origin were first ruled out. The side effects of corticosteroid use include opportunistic infections and vessel constriction, which may exacerbate gastric ischemia from causes other than HSP.

Endoscopic findings associated with HSP include redness, swelling, petechiae, submucosal hemorrhage, purpura, erosions and ulceration $(3,8)$. The small intestine, particularly the second portion of the duodenum, is the most frequently involved site (8). As the diagnostic yield of the endoscopic biopsy is low, the diagnosis of GI involvement of HSP depends on endoscopic findings and clinical features (9). Although the patient's skin biopsy exhibited leukoclastic vasculitis, analysis of the endoscopic biopsies of the duodenum and stomach was insufficient for a diagnosis of vasculitis.

Systemic corticosteroid is used to treat various diseases, including allergic disease (asthma, atopic dermatitis, and interstitial lung disease), hematologic diseases (lymphoma, leukemia and multiple myeloma), immunologic disorders (systemic lupus erythematosus and rheumatoid arthritis) and 

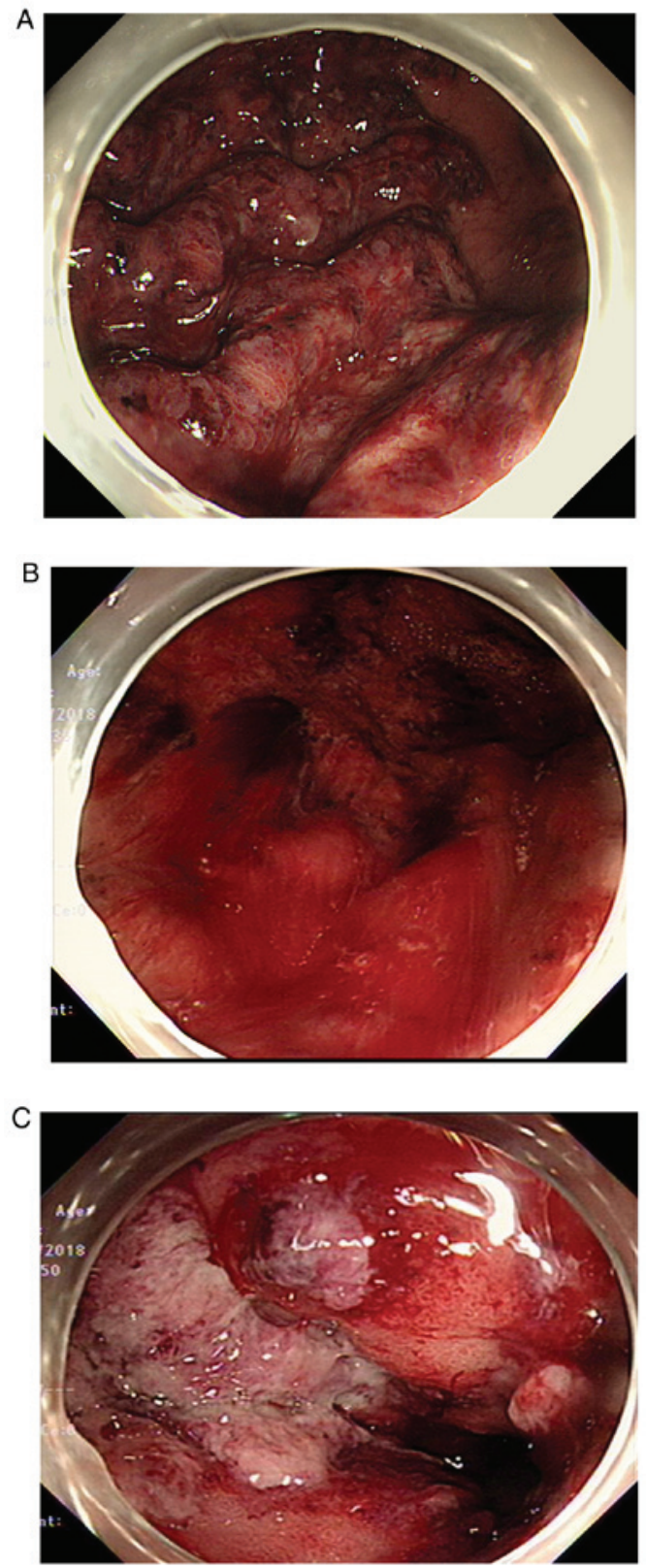

Figure 2. Endoscopic image revealing diffuse bleeding from stomach and duodenum. (A) Diffuse mucosal friability, pethechiae and purpura at the corpus. (B) Diffuse blood oozing was noted at the corpus. (C) Linear, active duodenal ulcer at bulb.

GI disease (inflammatory bowel disease and autoimmune hepatitis) (10). Corticosteroid is also the first-line treatment for HSP (6). However, the response to corticosteroid depends on the site of involvement. Corticosteroid is effective for abdominal pain, the most common GI manifestation (11). Common GI manifestations of HSP are postprandial abdominal pain, nausea, vomiting and GI bleeding. Although hematemesis has been rarely reported, the treatment efficacy of corticosteroid for GI bleeding is favorable (5). In the present case, the patient's abdominal pain improved and there was no further bleeding after administration of corticosteroid. The patient did not undergo hemostasis for GI bleeding. At one week after corticosteroid treatment, the follow-up EGD revealed marked improvement in the petechiae and purpura in the stomach and the duodenal ulcer was completely healed.

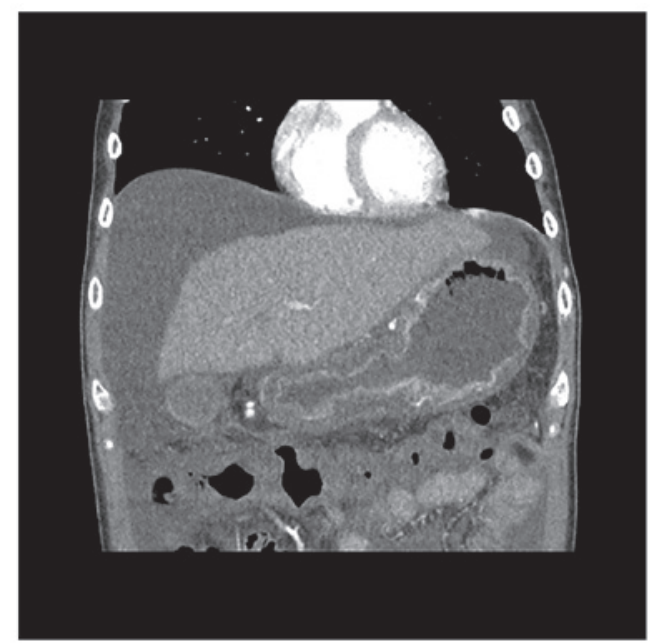

Figure 3. Abdominal computed tomography revealed diffuse edematous change at entire stomach with no occlusion of major vessels.
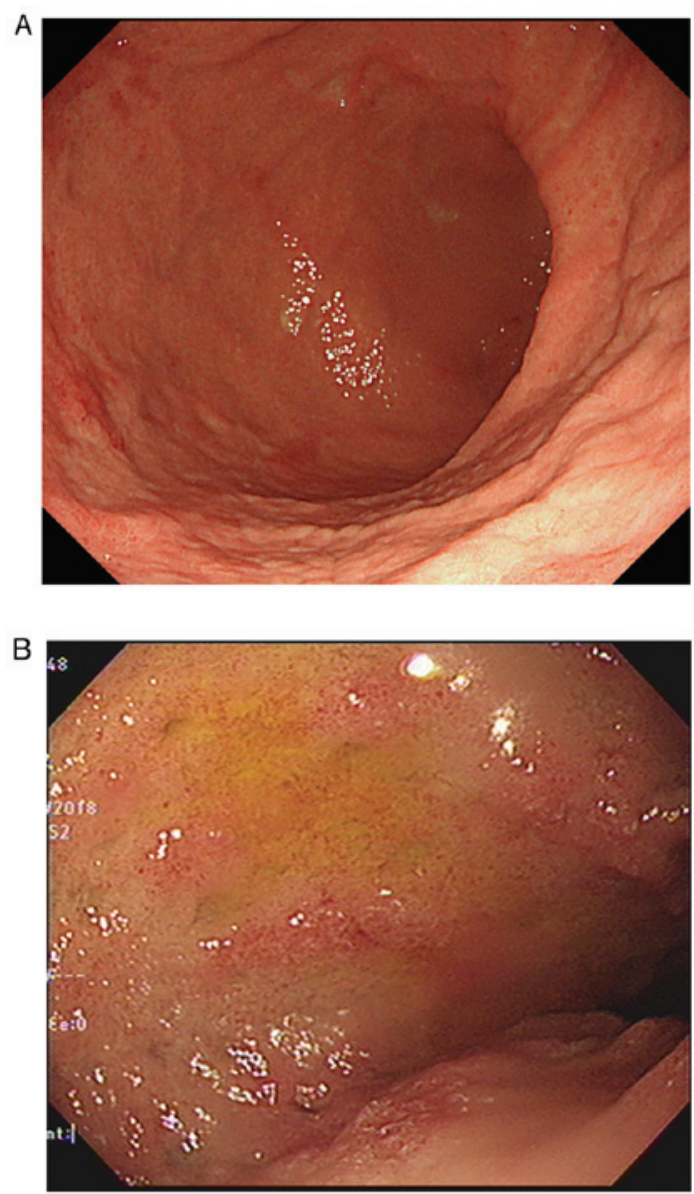

Figure 4. Follow-up endoscopy indicating regression of mucosal friability, purpura and ulcer. (A) Regression of mucosal friability and purpura. (B) Completely healed duodenal ulcer.

In conclusion, the present study reported on a rare case of acute exacerbation and rapid recovery of HSP manifested as massive gastric mucosal damage. This case exhibited a favorable response to corticosteroid used to treat the rapid progression of massive gastric mucosal damage complicated by HSP. 


\section{Acknowledgements}

Not applicable.

\section{Funding}

No funding was received.

\section{Availability of data and materials}

The datasets used and/or analyzed during the present study are available from the corresponding author on reasonable request.

\section{Authors' contributions}

TGG contributed to the analysis of data and drafting the manuscript. JHK contributed to data analysis and revised the manuscript critically for important intellectual content. All authors contributed to the literature search, and read and approved the final version of the manuscript.

\section{Ethics approval and consent to participate}

The study was approved by the Ethics Committee of the Incheon St. Mary's Hospital (Incheon, Republic of Korea). The patient provided written informed consent.

\section{Patient consent for publication}

The patient gave written informed consent for the investigations that were performed (blood tests and biopsy) and for the publication of medical data and images for scientific purposes.

\section{Competing interests}

The authors declare that they have no competing interests.

\section{References}

1. Saulsbury FT: Clinical update: Henoch-Schönlein purpura. Lancet 369: 976-978, 2007.

2. Babian M, Nasef S and Soloway G: Gastrointestinal infarction as a manifestation of rheumatoid vasculitis. Am J Gastroenterol 93: 119-120, 1998.

3. Ebert EC: Gastrointestinal manifestations of Henoch-Schönlein purpura. Dig Dis Sci 53: 2011-2019, 2008.

4. Lippl F, Huber W, Werner M, Nekarda H, Berger H and Weigert N: Life-threatening gastrointestinal bleeding due to a jejunal lesion of Henoch-Schönlein purpura. Endoscopy 33: 811-813, 2001.

5. Nam EJ, Kim GW, Kang JW, Im CH, Jeon SW, Cho CM, Jeong JY, Park JY, Jang YJ and Kang YM: Gastrointestinal bleeding in adult patients with Henoch-Schönlein purpura. Endoscopy 46: 981-986, 2014.

6. Audemard-Verger A, Terrier B, Dechartres A, Chanal J, Amoura Z, Le Gouellec N, Cacoub P, Jourde-Chiche N, Urbanski G, Augusto JF, et al: Characteristics and management of IgA vasculitis (Henoch-Schonlein) in adults: Data from 260 patients included in a french multicenter retrospective survey. Arthritis Rheumatol 69: 1862-1870, 2017.

7. Tang SJ, Daram SR, Wu R and Bhaijee F: Pathogenesis, diagnosis, and management of gastric ischemia. Clin Gastroenterol Hepatol 12: 246-252, 2014.

8. Esaki M, Matsumoto T, Nakamura S, Kawasaki M, Iwai K, Hirakawa K, Tarumi K, Yao T and Iida M: GI involvement in Henoch-Schönlein purpura. Gastrointest Endosc 56: 920-923, 2002.

9. Gong EJ, Kim do H, Chun JH, Ahn JY, Choi KS, Jung KW, Lee JH, Choi KD, Song HJ, Lee GH, et al: Endoscopic findings of upper gastrointestinal involvement in primary vasculitis. Gut Liver 10: 542-548, 2016

10. Liu D, Ahmet A, Ward L, Krishnamoorthy P, Mandelcorn ED, Leigh R, Brown JP, Cohen A and Kim H: A practical guide to the monitoring and management of the complications of systemic corticosteroid therapy. Allergy Asthma Clin Immunol 9: 30, 2013.

11. Weiss PF, Klink AJ, Localio R, Hall M, Hexem K, Burnham JM, Keren R and Feudtner C: Corticosteroids may improve clinical outcomes during hospitalization for Henoch-Schönlein purpura. Pediatrics 126: 674-681, 2010.

This work is licensed under a Creative Commons Attribution-NonCommercial-NoDerivatives 4.0 International (CC BY-NC-ND 4.0) License. 